\title{
Synthesis and analysis of cupper nanoparticles produced by laser
}

\author{
Mu'ataz S. Al Hassan' ${ }^{1}$ Fatima Ghali² \\ ${ }^{12}$ AlAyen University, Thi-Qar, Iraq. \\ muatazsalman1983@gmail.com¹, Fatema@alayen.edu.iq ${ }^{2}$
}

Article History: Received: 11 October 2020; Accepted: 27 December 2020; Published online: 8 February 2021

Abstract: Cupper nanoparticles are generated by using Nd:YAG laser nanosecond pulses using the liquid laser ablation technique. Nd:YAG of 6 and 10 nanoseconds pulse length with variable energy in the range are the two systems used (700$760 \mathrm{~mJ}$ ). The formation of cupper nanoparticles has been abolished using TEM with identical size distribution It has also been observed that when identical laser parameters are used, the average nanoparticles sizes of 80 and $120 \mathrm{~nm}$ for cupper are used.Furthermore, to approximate the temperature distributions for both cupper nanoparticles, the theoretical Mie-Gans model was used. Another factor that has been found is that the maximum temperature for gold nanoparticles is between $(1000 \mathrm{~K})$ and $(1400 \mathrm{~K})$, especially for the preparation of nanoparticles in the presence of $10 \mathrm{~ns}$ Nd:YAG.

Keywords: Nd:YAG laser, Cupper nanoparticles, TEM

\section{Introduction}

The word nanoscience is characterized as a range of technologies and developments that rely on nanoscale physical, chemical, and biological phenomena ranging from approximately 1 to 100 nanometers[1]. In general terms, nanomaterials are developed using two approaches: bottom-up and top-down[2].Nanomaterials are, however, differentiated into zero, one, two and three-dimensional nanostructures[3,4]. Nanomaterial methods of synthetization are either chemical or physical.Physical methods rely on wire technique, physical vapor deposition, thermal evaporation, sputtering deposition, deposition of chemical vapor and laser ablation, while there are two forms of laser ablation for the creation of NPs using laser ablation: dry and wet.In addition, Pulse Laser Ablation in Liquid (PLAL) requires the contact between the laser and the target, which can also be used to create nanoparticles with colloidal suspension[5]. PLAL is defined as a one-step top-down preparation technique for nanoparticles[6]. The key benefits of this method are 1- The ability to manufacture different kinds of nanomaterials, such as metals, noble metals, semiconductors, nanoalloys, oxides, magnetic and core shell nanostructure[7], 2- No need for vacuum equipment[8], 3- The aggregation can be regulated by surfactant molecules[9]. It is possible to draw a mathematical model based on the previous interaction.

$$
\left.\mathrm{T}(\mathrm{t}):=\frac{2 \cdot \mathrm{Fo}}{\mathrm{k}}\left[(\alpha \cdot \mathrm{t})^{\frac{1}{2}} \frac{\mathrm{e}^{-\left[\frac{\mathrm{z}}{2 \cdot(\alpha \cdot \mathrm{t} 1)^{\frac{1}{2}}}\right]}}{\frac{1}{\pi^{2}}-\frac{\mathrm{z}}{2 \cdot(\alpha \cdot \mathrm{t} 1)^{\frac{1}{2}}}\left[1-1-\left(\mathrm{a} \cdot \mathrm{b}+\mathrm{A} \cdot \mathrm{b}^{2}+\mathrm{C} \cdot \mathrm{b}^{3}\right) \mathrm{e}\right.}-\left[\frac{\mathrm{z}}{2 \cdot(\alpha \cdot \mathrm{t} 1)^{\frac{1}{2}}}\right]^{2}\right]\right]
$$

Where $\mathrm{T}(\mathrm{t})$ : Temperature $(\mathrm{K})$,Fo : Surface absorbed power W/m 2 P K : Thermal conductivity W/m.K , $\alpha$ :Thermal diffusivity mP2 sP-1, P t : Time s , z : Depth m, t1 : Time power off s and a,A1,C,b : Constant [10].

\section{Experimental}

Two laser systems are used for the ablation phase in this analysis; Nd:YAG. Nd:YAG laser produces 1064 $\mathrm{nm}$ pulses of an energy per pulse up to $1000 \mathrm{~mJ}, 10 \mathrm{~ns}$ pulse duration with a maximum repetition equal to $6 \mathrm{~Hz}$, while the second has an energy of $850 \mathrm{~mJ}$ and a pulse duration of 6 ns with a maximum repetition rate of 10 Hz.The focused Nd-YAG beam process is conducted using a $10 \mathrm{~cm}$ focal length lens on the cupper target. There is a very high purity of cupper plates; it is around (99). The plates should be polished, washed with ethanol and purified water, and broken into small parts to conform with the experimental procedure..Another significant note is that in this work, DDDW (Double Distilled and Deionized Water) is required to prepare all samples and solutions. To study the geometry of the prepared nanoparticles, scale and size distribution.TEM is used by morphological inquiry. In order to analyze the aggregation of the nanoparticles in the colloidal suspension, Zeta potential tests are also used.

\section{Experimental Details}


The temperature distribution of cupper nanoparticles was determined using the convolution of the distribution of size with the theory of heat flow as follows.

$$
\mathrm{T}(\mathrm{r} 1):=\int_{\mathrm{r}}^{\mathrm{ro}} \mathrm{N} \cdot \mathrm{D}(\mathrm{r} 1) \cdot\left[\frac { \mathrm { 2 } \cdot \frac { \mathrm { P } \cdot ( 1 - \mathrm { rf } ) \cdot \mathrm { r } 1 } { \mathrm { 6 } \cdot 1 0 ^ { 3 } \cdot \mathrm { v } ( \mathrm { r } 1 ) } } { \mathrm { k } } \left[(\alpha \cdot \mathrm{t})^{\frac{1}{2}} \frac{\mathrm{e}^{-\left[\frac{\mathrm{z}}{2 \cdot(\alpha \cdot \mathrm{t})^{\frac{1}{2}}}\right]^{2}}}{\frac{1}{\pi^{2}}}-\frac{\mathrm{z}}{2 \cdot(\alpha \cdot \mathrm{t})^{\frac{1}{2}}}\left[1-1-\left(\mathrm{a} \cdot \mathrm{b}+\mathrm{A} 1 \cdot \mathrm{b}^{2}+\mathrm{C} \cdot \mathrm{b}^{3}\right) \mathrm{e}\left[\begin{array}{c}
\mathrm{z} \\
\left.2 \cdot(\alpha \cdot \mathrm{t})^{\frac{1}{2}}\right]
\end{array}\right]^{2}\right] \mid d \mathrm{dr}\right.\right.
$$

Fig 1 and 2, Fig 1 and Fig 2 demonstrate the relationship between the temperature and the radius of nanoparticles for cupper nanoparticles in the solution of equations 1 and 2.

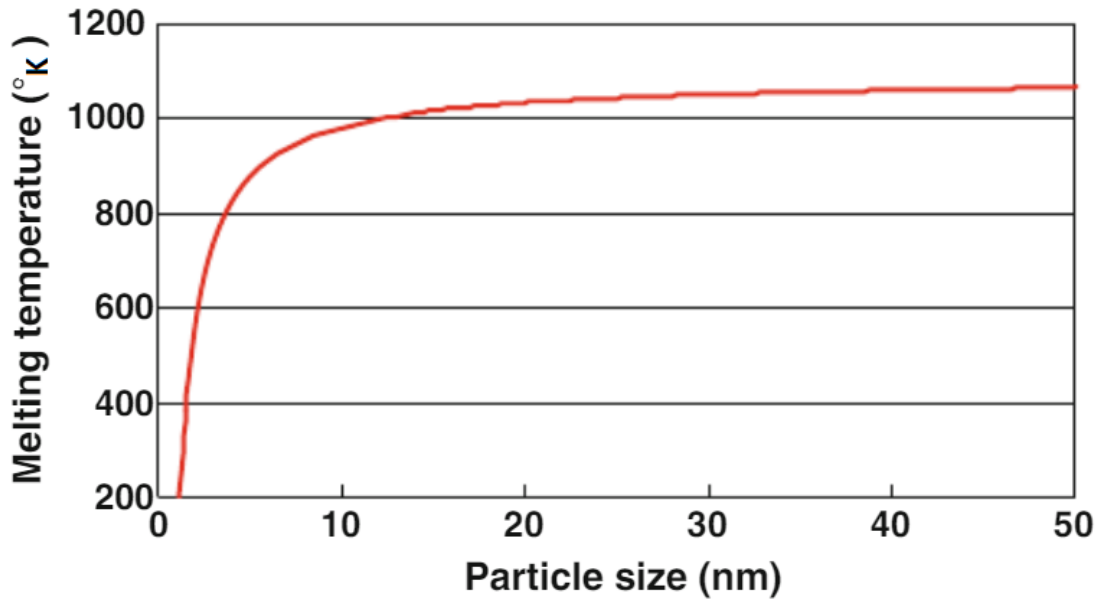

Figure (1) :The relationship between temperature and radius for laser nanoparticles has a pulse length of 10 $\mathrm{ns}$ for $740 \mathrm{~mJ}$ for nanoparticles of the cupper

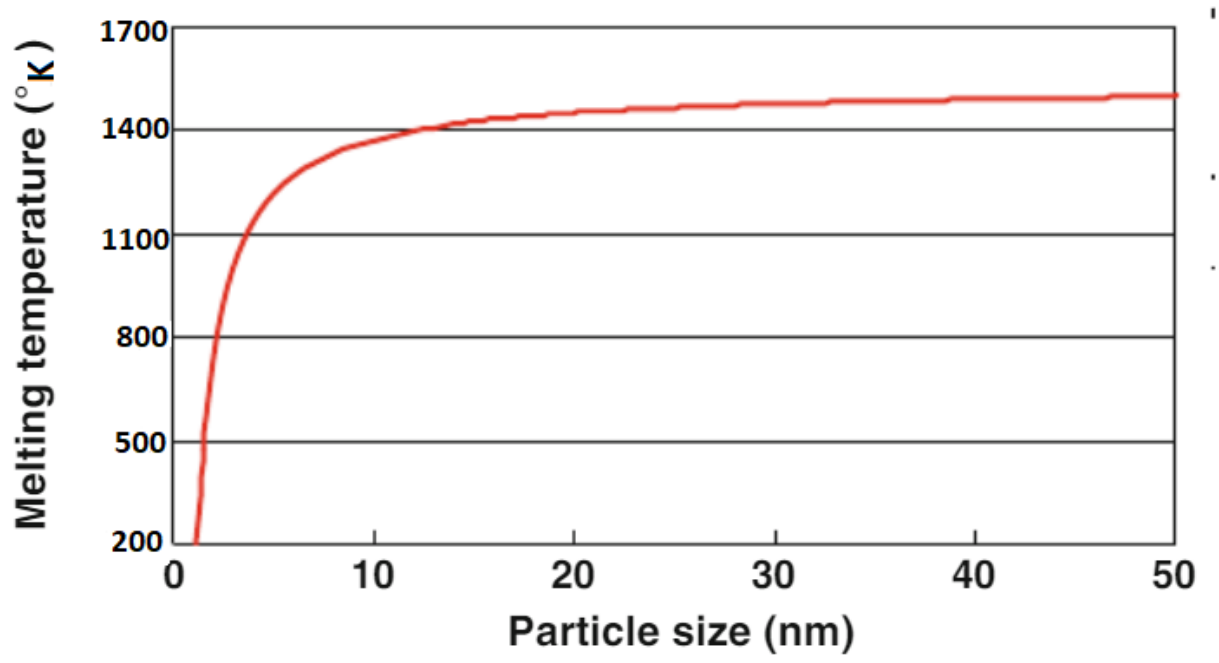

Figure (2):Temperature and radius of the laser forcuppernanoparticles of $6 \mathrm{~ns}$ and $740 \mathrm{~mJ}$ pulse length.A comparison of the theoretical results achieved for the lasers of both $10 \mathrm{~ns}$ and $6 \mathrm{~ns}$

Table 1: Comparison of the Au NPs produced by $1064 \mathrm{~nm}$ with pulse durations of 6 and $10 \mathrm{~ns}$ Duration of the laser pulse Molten depth $(\mu \mathrm{m})$ Maximum temperature (Co )

\begin{tabular}{|l|l|l|}
\hline Laser pulse duration & Molten depth $(\mu \mathrm{m})$ & Maximum temperature $\left(\mathrm{C}^{\circ}\right)$ \\
\hline $6 \mathrm{~ns}$ & 5 & 1400 \\
\hline $10 \mathrm{~ns}$ & 7 & 1000 \\
\hline
\end{tabular}

The Table indicates that

The temperature increases by $900 \mathrm{~K}$ as the laser pulse length falls from $10 \mathrm{~ns}$ to $6 \mathrm{~ns}$. 


\section{Experimental Results:}

TEM images of cupper $(\mathrm{Cu})$ nanoparticles formed by laser ablation with a pulse length of $6 \mathrm{~ns}$ and a power of 700 and $720 \mathrm{~mJ}$ are shown in figures $3 \mathrm{~A}$ and $3 \mathrm{~B}$, respectively.

The sizes of $\mathrm{c}$ are found to be due to high aggregation of small nanoparticles formed by higher energy,The dimensions of cupper nanoparticles have been found to increase by increasing laser energy.

The formation of small nanoparticles is due to a decrease in the temperature and vapor pressure produced, resulting in the release of small cupper nanoparticles.

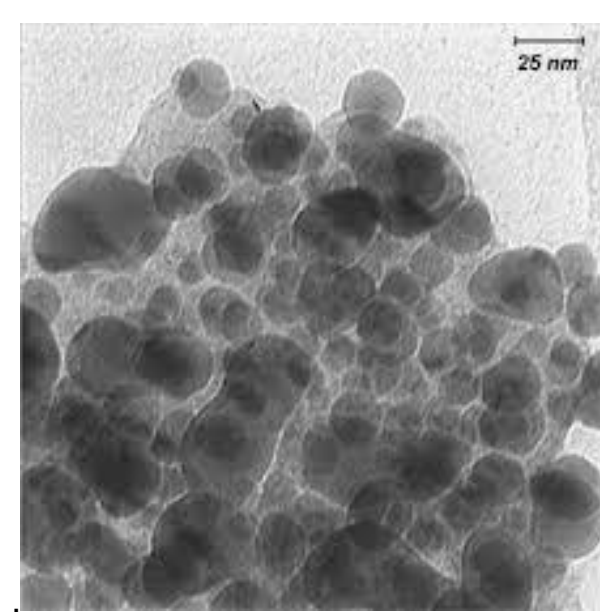

(A)

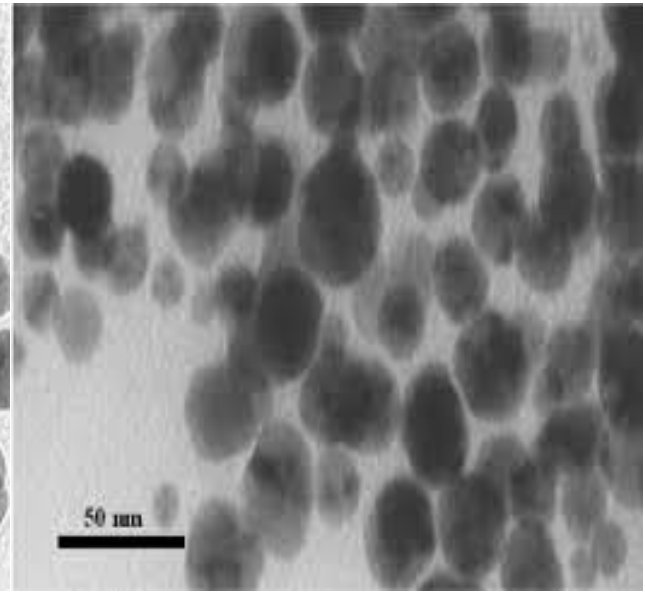

(B)

Fig 3 TEM images of cupper namoparticles for (a) 700mJ for $6 \mathrm{~ns}$ pulse duration

Using the histogram at the cuppa Fig 4. The size of nanoparticles for the distribution of cuppers (the homogeneous distribution) is due to Laser coefficient of absorption of high $\mathrm{Cu}(1064 \mathrm{~nm})$. This also induces temperature and vapor pressure, resulting in the subsequent ejection of small $\mathrm{Cu}$ nanoparticles.

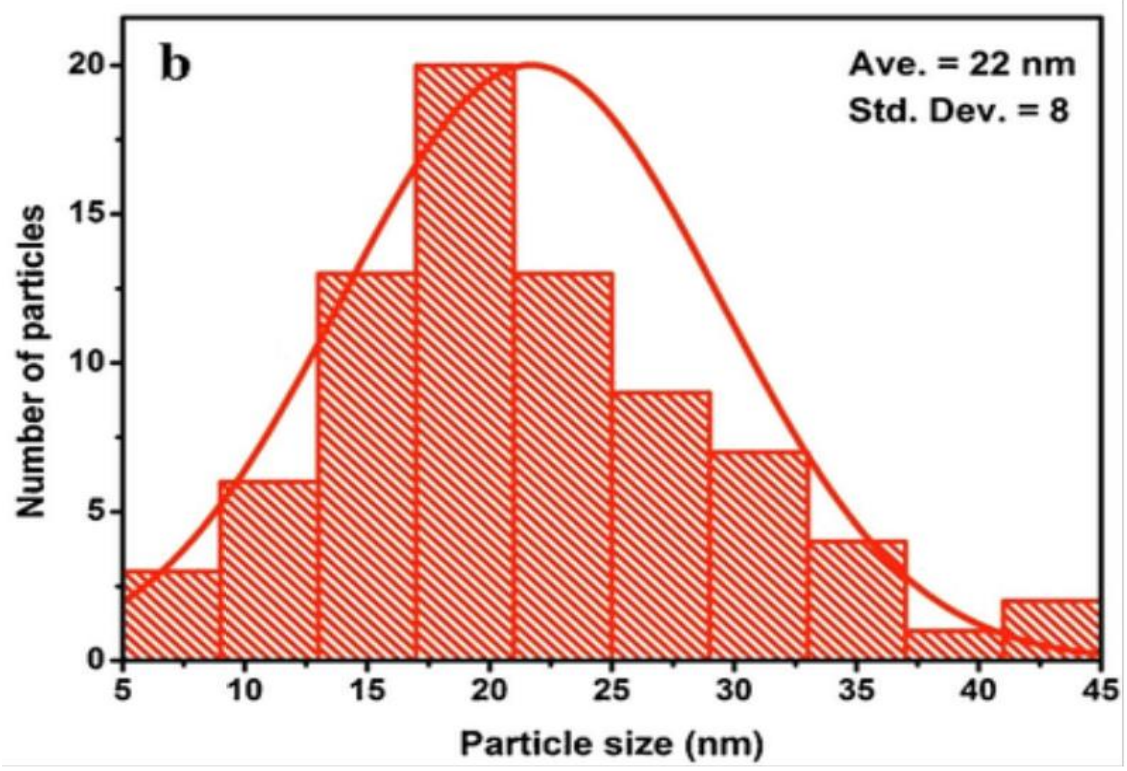

Fig 4 TEM histogram for cupper nanoparticles prepared with a pulse length of 700mJ and $10 \mathrm{~ns}$ of energy.

The absorption spectra of $\mathrm{Cu}$ nanoparticles UV-Vis are shown in Figure 5 (A and B), respectively. For nanoparticles prepared by (700 and 760) $\mathrm{mJ} /$ pulse with a $1064 \mathrm{~nm} \mathrm{Nd:YAG} \mathrm{laser,} \mathrm{all} \mathrm{spectra} \mathrm{were} \mathrm{measured.} \mathrm{The}$ peaks of UV-Vis spectra prepared with $700 \mathrm{~mJ}$ observed at $(429 \mathrm{~nm})$ of cupper nanoparticles indicate that the principal size was $(50 \mathrm{~nm})$. When the cupper particles are prepared with laser energy of $760 \mathrm{~mJ}$, this peak shifts 
to $(414 \mathrm{~nm})$. Due to the high temperature produced, this could be attributed to the effect of smaller nanoparticles being formed.

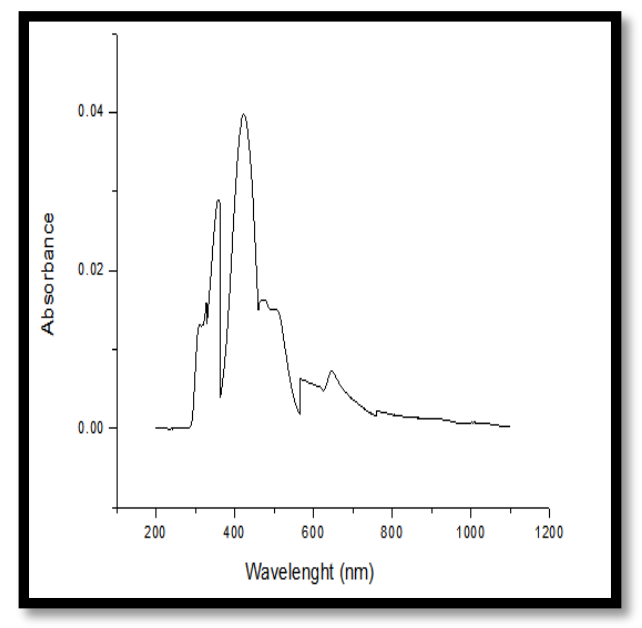

(A)

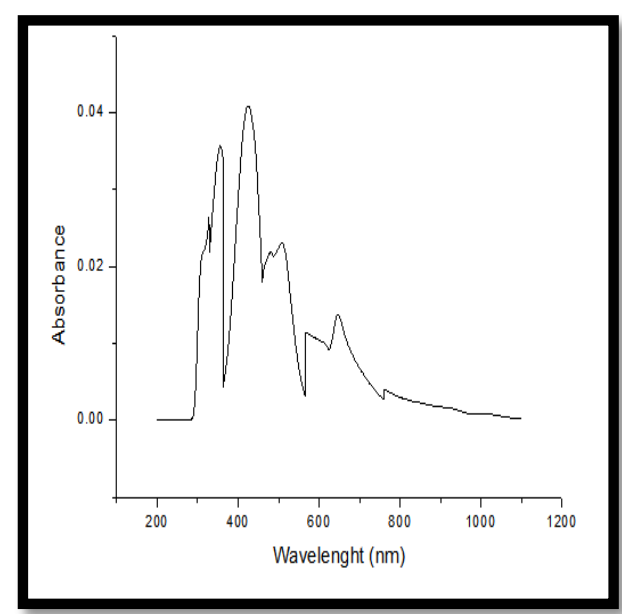

(B)

Fig 5. UVVIS absorption spectra of a solution of cupper nanoparticles primed by 200 laser energy pulses (A) $700 \mathrm{~mJ}$ and (B) $760 \mathrm{~mJ}$.

The existence of a single plasmon surface peak suggested that in the case of ellipsoidal particles, the nanoparticles produced are almost spherical while the absorption spectrum will have two plasmon peaks.The stability of the manufactured nanoparticles by their values was usually considered to be stable between

$+30 \mathrm{mV}$ and less than $-30 \mathrm{mV}$ and was compatible with the requirements for Z-potential inspection values. The principal value of the Z-potential in the cupper was- $49.42 \mathrm{mV}$.

\section{Conclusions:}

Stable, uniform-sized cupper nanoparticles can be predestined by laser liquid ablation. For cupper nanoparticles, temperature distributions of greater magnitude have been observed. If shorter laser pulses are used, the overall magnitude would be super-fat. The collection of the experimental size distribution data in the theoretical model offers a better understanding of the development of nanoparticles.

\section{References}

A. Mu,ataz S. Al Hassan, "Synthesis and modeling of temperature distribution for Si and Ag nanoparticels produced using Nd:YAG laser," J. Baghdad Univ.

B. P. Kazakevich, A. Simakin, and G. a. Shafeev, "Formation of periodic structures by laser ablation of metals in liquids," Appl. Surf. Sci., vol. 252, no. 13 SPEC. ISS., pp. 4457-4461, 2006.

C. R. Psaro, M. Guidotti, M. Sgobba, and B. Sketches, Nanosystems, vol. II. 2009.

D. D. A.Vladimir M. Mirsky, NEW ANALYTICAL Fredy Kurniawan NEW ANALYTICAL Presented by Fredy Kurniawan. 2008.

E. T. Tsuji, D.-H. Thang, Y. Okazaki, M. Nakanishi, Y. Tsuboi, and M. Tsuji, "Preparation of silver nanoparticles by laser ablation in polyvinylpyrrolidone solutions," Appl. Surf. Sci., vol. 254, no. 16, pp. 52245230,2008

F. A. Ali and D. Raouf, "Preparation of Silver Nanoparticles by Pulsed Laser Ablation in Liquid Medium," vol. 29, no. 15, p. 10, 2011.

G. R. Nakajima, X. Zhu, and T. Ishigaki, "Generation of Alumina nanoparticles by a nanosecond laserinduced liquid plasma in water," Ispc_20, pp. 2-5, 2011.

H. J. S. Golightly, J. S. Golightly, and N. Winograd, "Formation and characterization of nanoparticles via laser ablation in solution", no.1, May. 2007.

I. D. Werner, S. Hashimoto, T. Tomita, S. Matsuo, and Y. Makita, "In-situ spectroscopic measurements of laser ablation-induced splitting and agglomeration of metal nanoparticles in solution,” J. Phys. Chem. C, vol. 112, no. 43, pp. 16801- 16808, 2008. 
J. W. M. S. J. Mazumder, "Laser Material Processing" Fourth Edition, vol. 33. 2012. 